\title{
People, Technology, and Complex Work in Healthcare
}

\author{
Silvia Gilardi \\ Universitá di Milano \\ silvia.gilardi@unimi.it \\ Shawna J Perry \\ Virginia Commonwealth University \\ sperry4@mcvh-vcu.edu \\ Stephanie Wilson \\ City University London \\ r.wears@imperial.ac.uk
}

\author{
Chiara Guglielmetti \\ Universitá di Milano \\ chiara.guglielmetti@unimi.it \\ Gabriella Pravettoni \\ Universitá di Milano \\ gabriella.pravettoni@unimi.it \\ Robert L Wears \\ University of Florida; Imperial College London \\ wears@ufl.edu; r.wears@imperial.ac.uk
}

\begin{abstract}
Motivation - Healthcare are rapidly replacing manual with computerized artefacts for many reasons, but the impact on users of the technology is often assumed to be neutral. Approach - Three presentations of the increasingly more intensive use of computer technology in healthcare will be discussed by panelists and audience to explore the problem space. A closing presentation will highlight issues in designing technology that is both usable and useful to frontline workers. Originality/Value - There is great pressure to speed up the introduction of technology in healthcare, but a "rush to implementation" risks serious design problems. Take away message - Technological artefacts are introduced to meet many needs, but if users' needs are not taken into account, the technology may founder or be subverted.
\end{abstract}

Keywords

Health care, information technology, human-computer interaction, task-artefact cycle

\section{INTRODUCTION}

Healthcare workers operate in settings that possess many of the characteristics associated with naturalistic decisionmaking: complexity, uncertainty, ambiguity, goal conflicts, shifting priorities, time pressure, and multiple agents. They use informational artefacts to support them in this work, both at an individual level (for example, aids to prospective memory) and on a workgroup level (coordinating work that is distributed across time, space, and workers, both within and among different professional disciplines). These uses occur throughout clinical work, but may be easily observed at certain points in time, such as transfers of responsibility for patient care. For example, workers going off-shift must transfer their authority, responsibility, and situational knowledge, assessments and plans about patients and their conditions, work in progress and remaining to be done, stance towards changes in plan, etc, to members of the on-coming shift. A variety of artefacts have been used to support this activity; they can be roughly grouped into three categories of increasing complexity: general purpose artefacts used in special ways (eg, paper notes, personal computers); manual, special purpose artefacts (eg, shared display boards); or computer-based, special purpose artefacts (eg, clinical information systems).

\section{PANELISTS AND THEMES}

This panel will present observations of complex work from a variety of clinical settings. It will focus regarding how clinical workers use these different technologies in varying ways to support their work and in particular, their functioning as a joint cognitive system. The presentations will follow a roughly increasing order of technological 'intensity'.

\section{Rigidities and workarounds}

A psychologist / social psychologist with research interests in decision-making in complex settings will discuss ethnographic observations in a medium sized emergency department (ED) in northern Italy, focusing on workarounds that triagists use to overcome limits and rigidities of the personal computer mediated communication system adopted in the ED (Pravettoni, 2008). Because there is a shared perception that the computer-based artefact loses subtle nuances of the triagist's perspective, work practice observations have highlighted the triagists' activation of verbal, direct, synchronous communication to direct physicians' attention to crucial information to facilitate management. This informal practice produces both risks and benefits to organizational goals. (Professor Gabriella Pravettoni). 


\section{Manual status boards}

An emergency physician and researcher in patient safety will present how workers in EDs and hospital wards in the US and UK have spontaneously developed a shared artefact to support both individual and collective work. These take the form of large dry erase boards, originally used for tracking patient locations but over time extended to include a great deal of additional functionality (R L Wears, Perry, Salas, \& Burke, 2005). Because they have been developed directly by frontline workers, with almost no influence from management or external regulators, they offer a window into the issues that workers find difficult and important in their work. (Dr Shawna Perry).

\section{Computerized status boards}

An emergency physician and safety researcher with a background in software engineering, while discussing some implications of computerizing ED status boards. For a variety of reasons, the manual "whiteboards" described above are gradually being supplanted by computer-based information systems. These computerized systems emulate the form of the manual whiteboards but often have failed to capture some of their important functionalities. This presentation will describe a before-after study of the change from manual to computerized artefacts and the positive and negative effects of the change on clinical work (Pennathur, Bisantz, Fairbanks, Perry, Zwemer, \& Wears, 2008; Robert L Wears, Perry, Wilson, Galliers, \& Fone, 2007). (Dr Robert Wears)

\section{Designing usable technology}

A researcher interested in design of usable technology and in studying the use of technology, particularly in healthcare settings will build on the observation that it is increasingly the case that specialized computer-based artefacts are being introduced to support critical coordinative and communicative aspects of clinical work. This presentation will report recent efforts to design these kinds of technological artefacts to support clinical handover and how these efforts have been informed by ethnographic studies in varied healthcare settings. The studies have focused not on the details of the information that is handed over but on providing holistic accounts of the work. They have highlighted the importance for handover artefacts of promoting good situation awareness, through mechanisms such as representing the evolution of the situation, clinical and non-clinical decisions and explanations for those decisions (Robert L Wears et al., 2007; Wilson, Galliers, \& Fone, 2005). The presentation will draw on these experiences to consider the challenges inherent in moving from an "as is" situation (which may or may not be a good one) to one that embraces new technological opportunities without impeding the work. (Professor Stephanie Wilson).

\section{CONCLUSION}

There are many benefits to be had from greater computerization in healthcare, but the effect of this introduction on front-line workers is often assumed to be neutral. Exploring the latent functionality and cognitive properties of existing artefacts should usefully inform the design of new technologies so that negative impacts on users can be minimized or avoided.

\section{ACKNOWLEDGMENTS}

Stephanie Wilson's research is funded by the UK Engineering and Physical Sciences Research Council (EPSRC), grant number: (EP/D078636/1).

Robert Wears's and Shawna Perry are funded by the US Agency for Healthcare Research and Quality (1R18 HS017902-01 and 1P20 HS017141-01)

\section{REFERENCES}

Pennathur, P. R., Bisantz, A. M., Fairbanks, R. J., Perry, S. J., Zwemer, F. L., \& Wears, R. L. (2008). Cognitive Artifacts in Transition: An Analysis of Information Content Changes Between Manual and Electronic Patient Tracking Systems. Paper presented at the Human Factors and Ergonomics Society 52nd Annual Meeting.

Pravettoni, G. (2008). Decision process and situation awareness in emergency departments. Paper presented at the 9th International Symposium on Human Factors in Organizational Design and Management.

Wears, R. L., Perry, S. J., Salas, E., \& Burke, C. S. (2005). Status boards in emergency departments: support for shared cognition. In R. Tartaglia, S. Bagnara, T. Bellandi \& S. Albolino (Eds.), Healthcare Systems, Ergonomics and Patient Safety (pp. 273 - 280). Leiden, NE: Taylor \& Francis.

Wears, R. L., Perry, S. J., Wilson, S., Galliers, J., \& Fone, J. (2007). Emergency department status boards: user-evolved artefacts for inter- and intra-group coordination. Cognition, Technology \& Work, 9(3), 163-170.

Wilson, S., Galliers, J., \& Fone, J. (2005). Medical handover: a study and implications for information technology. In R. Tartaglia, S. Bagnara, T. Bellandi \& S. Albolino (Eds.), Healthcare Systems, Ergonomics, and Patient Safety (pp. 333 - 336). Leiden: Taylor \& Francis. 